\title{
Brainstem Tuberculoma Reactivated during Pregnancy and Postpartum
}

\author{
D. Kanikomo', M. Diallo', A. Tokpa ${ }^{2}$, I. Sango 3 , Y. Sogoba', M. S. Diarra ${ }^{4}$, \\ B. Sogoba1, M. Dama5, 0. Coulibaly5, M. Coulibaly', M. Diallo \\ ${ }^{1}$ Department of Neurosurgery, Teaching Hospital Gabriel Touré, Bamako, Mali \\ ${ }^{2}$ Department of Neurosurgery, Teaching Hospital of Bouaké, Bouaké, Côte d'Ivoire \\ ${ }^{3}$ Department of Radiology, Teaching Hospital Gabriel Touré, Bamako, Mali \\ ${ }^{4}$ Department of Neurosurgery, Luxembourg Hospital of Bamako, Bamako, Mali \\ ${ }^{5}$ Department of Neurosurgery, Mali Hospital, Bamako, Mali \\ Email:mdiallo5@gmail.com
}

How to cite this paper: Kanikomo, D., Diallo, M., Tokpa, A., Sango, I., Sogoba, Y., Diarra, M.S., Sogoba, B., Dama, M., Coulibaly, O., Coulibaly, M. and Diallo, M. (2022) Brainstem Tuberculoma Reactivated during Pregnancy and Postpartum. Open Journal of Modern Neurosurgery, 12, 1-8. https://doi.org/10.4236/ojmn.2022.121001

Received: September 29, 2021

Accepted: December 6, 2021

Published: December 9, 2021

Copyright (C) 2022 by author(s) and Scientific Research Publishing Inc. This work is licensed under the Creative Commons Attribution International License (CC BY 4.0).

http://creativecommons.org/licenses/by/4.0/

\begin{abstract}
Pregnancy, which is responsible for the decline in immunity, and the immediate postpartum period can lead to reactivation or worsening of tuberculosis. We report a case of a patient who consulted for neurological disorders in the context of a deterioration in general condition. The CT scan revealed a brainstem lesion which was successfully treated like a tuberculosis. However, reactivation has been observed in the postpartum period of a pregnancy contracted during anti-tuberculosis treatment. Further clinical improvement has been achieved with anti-tuberculosis treatment. Pregnancy and the immediate postpartum had led to a transient decline in immunity in part by decreasing in the inflammatory activity of type 1 helper $\mathrm{T}$ cells so that the fetus, which is a foreign body, was accepted by the maternal body. This decline in immunity during pregnancy and the immediate postpartum period due to immune reconstitution had been responsible for a high degree of vulnerability, usually characterized by a significant exacerbation of tuberculosis symptoms and an unfavorable course of disease.
\end{abstract}

\section{Keywords}

Tuberculosis, Central Nervous System, Reactivation, Pregnancy

\section{Introduction}

Around a quarter of the world's population has latent tuberculosis infection [1]. The reactivation of these latent infections in active tuberculosis is favored by even transient immunosuppression. The information found in the literature on 
the promoting role of pregnancy in the onset of tuberculosis is contradictory. For some author's pregnancy does not increase the risk of active tuberculosis and even go further to say that it reduces the risk of occurrence of active tuberculosis. Others, on the other hand, demonstrate the contrary. In a recent study, Jonsson J. et al. had shown a significant increase in risk of active TB during pregnancy and particularly during postpartum in women from high incidence countries. The increased risk of active TB postpartum has been interpreted as possibly due to delayed diagnosis, as symptoms of active TB can be mistaken for symptoms related to the pregnancy itself. The particular biological changes that occur in a woman's body during pregnancy and postpartum period increase the risk of tuberculosis at least twice, compared to women who are not pregnant [2] [3]. We report a case of reactivation in the postpartum period of a previously successfully treated brainstem tuberculoma.

\section{Observation}

A 30-year-old unemployed woman weighing $49 \mathrm{~kg}$ at the time of diagnosis complained in February 2018 of progressively worsening neurological disorders. She resided in a hamlet sharing the same courtyard with the goats and sheep. In her history, she reported episodes of fever with anorexia, a deterioration in general condition and a tuberculosis contagion with a member of her family living the same roof and being treated for pulmonary tuberculosis. The neurological examination carried out on admission on 04/17/2018 revealed Millard-Gubler syndrome (left peripheral facial paralysis associated with right hemiplegia sparing the face). Brain computed tomography objectified an intra-axial hypodense lesion with a ring contrast enhancement (cockade image) located in the pons slightly to the left (Figure 1 and Figure 2). The Mantoux tuberculin skin test

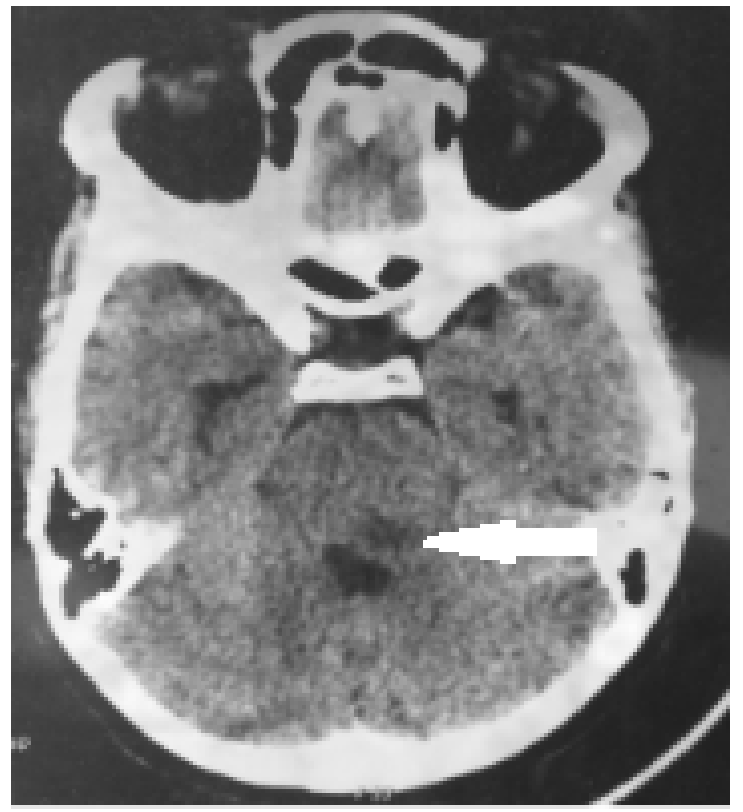

Figure 1. CT brain without contrast showing pontine hypodensity round focal lesion. 


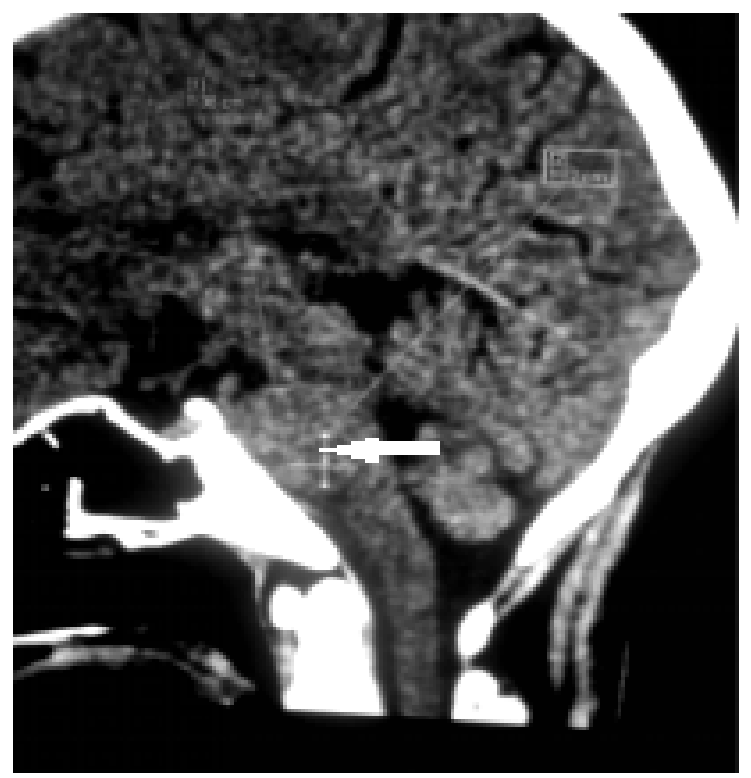

Figure 2. CT brain with contrast showing a moderate ring enhancement of a pontine round focal lesion.

was positive with an induration of $15 \mathrm{~mm}$. White blood cell count was normal but the patient had anemia with a hemoglobin level at $9.8 \mathrm{~g} / 100 \mathrm{ml}$. The level of C-reactive protein was normal (less than $5 \mathrm{mg} / \mathrm{l}$ ). Her HIV and toxoplasma serologies were negative. The diagnosis of brainstem tuberculoma was retained and anti-tuberculosis treatment, according to the regimen of 2 RHZE (2 months of rifampin isoniazid pyrazinamide ethambutol) followed by $10 \mathrm{PE}$ (10 months of pyrazinamide and ethambutol) was instituted. Vitamin B6, B9 and iron supplementation has been associated with anti-tuberculosis treatment.

Complete resolution of the neurological signs and weight gain were observed $(53 \mathrm{~kg}$ ) on examination in the third month of treatment. A follow-up CT was scheduled for the $12^{\text {th }}$ month of treatment before stopping multidrug therapy.

In the control examination on March 23, 2019, in the eleventh month of treatment, no complaints were noted and the physical examination was normal. The follow-up CT could not be performed before stopping treatment because of the finding of a pregnancy of 2 months. There was no MRI available. The anti-tuberculosis drugs were stopped after one year of treatment and at the same time, obstetric ultrasound showed an evolving monofetal pregnancy of 30 weeks, male and normal morphology. The pregnancy proceeded normally until term and the vaginal birth occurred without any particular problem.

In March 2020, approximately one year after stopping anti-tuberculosis treatment and 3 months after childbirth, the patient complained of the resumption of the clinical signs with fever and anorexia. Symptoms worsened in January 2021 with the gradual onset of right hemi-corporeal motor deficit, right upper limb tremors and dysarthria. A new brain computed tomography had revealed a spontaneously hyperdense mass with a moderate ring contrast enhancement, still located in the pons (Figure 3). 


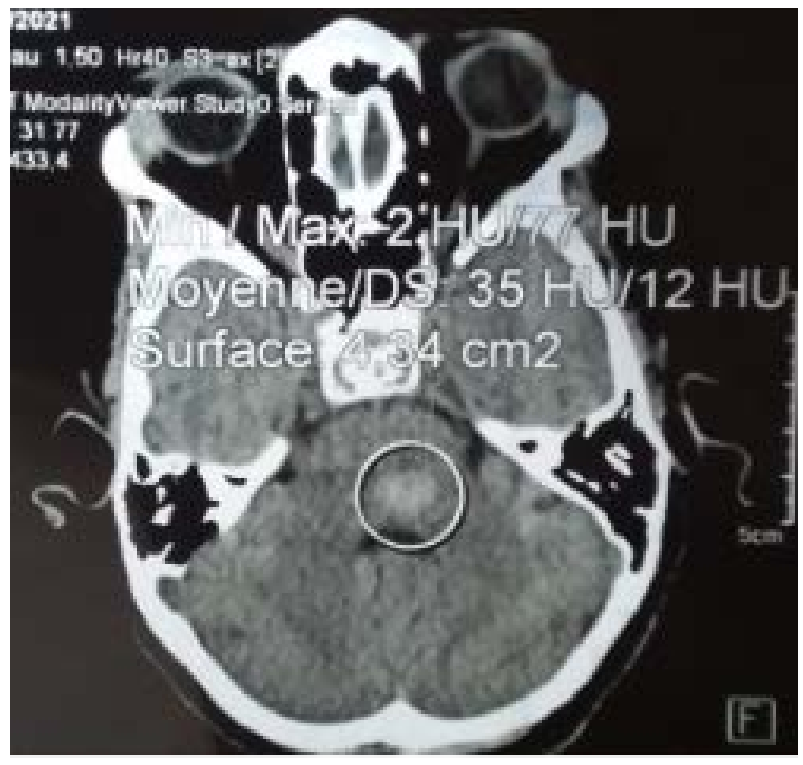

Figure 3. Axial contrast CT image showing moderate ring contrast enhancement.

The new Mantoux tuberculin skin test was negative and complete blood count was normal. The diagnosis of reactivation of the brainstem tuberculoma was made and the patient resumed anti-tuberculosis treatment with vitamin B6 supplementation and oral contraceptives. After two months of treatment, complete resolution of neurological signs was observed.

\section{Discussion}

The frequency of cerebral tuberculomas remains particularly high in developing countries where they remain an important localization of tuberculosis, reaching $5 \%$ to $40 \%$ of intracranial expansive processes [4] [5]. This can be explained by the difficulties of access to care, poor hygienic conditions, overcrowding and malnutrition, which are risk factors favoring the occurrence of tuberculosis [6]. All these conditions were met in our patent. Pregnancy and the immediate postpartum lead to a transient decline in immunity so that the fetus, who is a foreign body, is accepted by the maternal body. Thus, Simmons and Russels [7] in their experiments showed that a female who tolerates the conceptus (fetus, placenta and appendages) will reject the tissues of the fetus and then of any organ of her baby if it is transplanted after birth, within a period of transplant rejection after 14 to 17 days. This transient tolerogenesis is dependent on several factors: the major histocompatibility complex (MHC) specific to the fetal condition (HLAG), a decrease in the activity of NK cells (Natural Killer, non-B-non-T-lymphocyte) and reduced chemotactic possibilities of phagocytic cells. According to Ellis et al. [8], in humans, MHC molecules (HLA antigens) are localized at the so-called "extravillous" part of the placenta. These would only be monomorphic HLA antigens of a new type. The decrease in killer cells could be explained, according to Voisin [9], by the production of "facilitating" or "blocking" antibodies. 
These would then mask the histocompatibility antigens which would no longer be detected by the immunological cells of the mother. Such antibodies can in fact be eluted from placentas implanted in the uterus of a "producer" mouse. These "blocking" antibodies can mask other foreign bodies such as Mycobacterium tubrculosis. On the other hand, in the absence of anergizing disease in our patient, these blocking antibodies could be responsible for tuberculin anergy especially since the pre-pregnancy Mantoux tuberculin skin test was positive with an induration of $15 \mathrm{~mm}$. In contrast, humoral immunity remains normal during pregnancy. The B lymphocytes are not modified, the quantity of immunoglobulins is normal except for the IgG, which are reduced in the $3^{\text {rd }}$ trimester due to an increasing passage into the fetal circulation which partly explains the worsening of tuberculosis from the $6^{\text {th }}$ month of pregnancy. On the other hand, some maternal hormones contribute to the decline of immunity [10]. Thus progesterone decreases the innate immune response (macrophages, NK cells). Estradiol, in high doses, acts as a brake on NK-type immune cells. As for estriol, the real keystone of maternal immune tolerance during pregnancy, it is a hormone specifically and only expressed during gestation. Its effects are: increase in anti-inflammatory cytokines (IL-5), decrease in pro-inflammatory cytokines (TNF- $\alpha$, IFN- $\gamma$ ), decrease in $\mathrm{CD} 4+$ and $\mathrm{CD} 8+\mathrm{T}$ numbers, increase in autoimmune response. Several cases of worsening pregnancy-related tuberculosis have been reported. The particular biological changes that occur in a woman's body during pregnancy lead to a decrease in the inflammatory activity of type 1 helper $T$ lymphocytes. Mathad [11] recently showed that cultures of human placenta, whose viability was verified by assaying the secretion of chorionic gonadotropic hormone (hCG) also secreted factors that could block both the production of murine and human killer cells, and the growth of murine cell lines dependent on interleukin 2. The blocking effect was reversible by addition of excess IL-2. Finally, the placenta releases its receptors for the constant part of the antibodies, which, locally, modifies any antibody which binds to it into a blocking antibody, by inactivation of the part of this molecule which fixes the complement, essential for the lysis. According to Zenner [2] pregnant women are twice as likely to contract tuberculosis with a more subtle picture and more complications. This risk would be $27 \%$ of pulmonary locations according to the work of Pridie and Stradling [12]. In a cohort study of pregnant women, they noted an incidence of 15.4 cases of tuberculosis (all locations) per 100,000 pregnancies. These tuberculosis had occurred during pregnancy or in the first 6 months postpartum. In our patient, signs of worsening appeared in the $3^{\text {rd }}$ month postpartum. Other authors have reported cases of worsening in connection with pregnancy [13] [14]. During the immediate postpartum period, there is still a high degree of vulnerability due to immune reconstitution, usually characterized by a significant exacerbation of tuberculosis symptoms and an unfavorable course of the disease [11]. The treatment recommended by WHO is the same as that used for the general population with regard to medication and dosage, and most national guidelines now agree 
with this approach [14]. However, administration of pyrazinamide during pregnancy is not recommended by the United States Centers for Disease Control and Prevention, the American Thoracic Society, and the American Society of Infectious Diseases. In our case the pregnancy occurred in the second phase, which did not contain pyrazinamide. It is classically recommended the administration of vitamin B6 during pregnancy with a slightly higher dose of $25 \mathrm{mg} /$ day. The evolution was favorable with the disappearance of the neurological signs since the $2^{\text {nd }}$ month of treatment in our patient. The 2015 WHO report also reports that mortality has fallen by $47 \%$ since 1990 [15]. According to Awada [16], the rate of regression of tuberculoma undergoing antibacterial treatment is slow during the first month, accelerates between the $2^{\text {nd }}$ and the $5^{\text {th }}$ month, then slows down again after the $6^{\text {th }}$ month to lead to a total disappearance of any tuberculous image in the $11^{\text {th }}$ month. Thus, the duration of anti-tuberculosis treatment varies in the literature. However, many authors (British and American international recommendations and those of the Center of Disease Control) have recommended a duration of 9 to 12 months [17] [18]. This duration is usually accompanied by regression of tuberculomas. A longer period of 18 months to 3 years is recommended by some authors given the persistence of minimal lesions on imaging. This has led some therapists to continue treatment for longer periods, sometimes reaching 3 years [19] [20]. On the other hand, other authors have reported that stopping treatment at 12 months, despite the persistence of small lesions, did not worsen the patient's condition [21].

\section{Conclusion}

Pregnancy with the resulting reduced immunity and the postpartum period can reactivate tuberculoma, which is well controlled with multidrug therapy for tuberculosis. Practitioners should be aware of the unpredictable symptoms of tuberculosis during pregnancy. The occurrence of pregnancy during anti-tuberculosis treatment requires close monitoring and the duration of treatment may be discussed.

\section{Conflicts of Interest}

The authors declare no conflicts of interest regarding the publication of this paper.

\section{References}

[1] World Health Organization (2020) Global Tuberculosis Report 2020. World Health Organization, Geneva.

[2] Zenner, D., Kruijshaar, M.E., Andrews, N. and Abubakar, I. (2012) Risk of Tuberculosis in Pregnancy: A National, Primary Care-Based Cohort and Self-Controlled Case Series Study. American Journal of Respiratory and Critical Care Medicine, 185, 779-784. https://doi.org/10.1164/rccm.201106-1083OC

[3] Jonsson, J., Kühlmann-Berenzon, S., Berggren, I., et al. (2020) Increased Risk of Active Tuberculosis during Pregnancy and Postpartum: A Register-Based Cohort Study in Sweden. European Respiratory Journal, 55, Article ID: 1901886. 


\section{https://doi.org/10.1183/13993003.01886-2019}

[4] Ramamurthi, B., Ramamurthi, R. and Vasudevan, M. (1986) Changing Concepts in the Treatment of Tuberculomas of the Brain. Child s Nervous System, 2, 242-243.

[5] Gropper, M.R., Schulder, M., Duran, H.L. and Wolansky, L. (1994) Cerebral Tuberculosis with Expansion into Brainstem Tuberculoma: Report of Two Cases. Journal of Neurosurgery, 81, 927-931. https://doi.org/10.3171/jns.1994.81.6.0927

[6] Zein, M., Fletcher, P.S. and Mirghani, Z.M. (2000) Intracranial Tuberculoma. Saudi Medical Journal, 21, 196-199.

[7] Simmons, R.L. and Russel, P.S. (1962) The Antigenicity of Mouse Trophoblast Cells. Annals of the New York Academy of Sciences, 99, 717-724. https://doi.org/10.1111/j.1749-6632.1962.tb45356.x

[8] Ellis, S.A., Sargent, I.L., Redman, C.W.G. and McMichael, A.J. (1986) Evidence for a Novel HLA Antigen Found on Human Extravillous Trophoblast and a Choriocarcinoma Cell Line. Immunology, 59, 595-601.

[9] Voisin, G.A. and Chaouat, G. (1974) Demonstration, Nature and Properties of Maternai Antibodies Fuced on Placenta and Directed against Paterna! Alloantigens. Journal of Reproduction and Fertility, 21, 89-107.

[10] Robinsona, D.P. and Kleina, S.L. (2012) Pregnancy and Pregnancy-Associated Hormones Alter Immune Responses and Disease Pathogenesis. Hormones and Behavior, 62, 263-271. https://doi.org/10.1016/j.yhbeh.2012.02.023

[11] Mathad, J.S. and Gupta, A. (2012) Tuberculosis in Pregnant and Postpartum Women: Epidemiology, Management, and Research Gaps. Clinical Infectious Diseases, 55, 1532-1549. https://doi.org/10.1093/cid/cis732

[12] Pridie, R. and Stradling, P. (1961) Management of Pulmonary Tuberculosis during Pregnancy. British Medical Journal, 2, 78-79. https://doi.org/10.1136/bmj.2.5244.78

[13] Martin, L., Cropley, I., Wilkinson, R. and Davidson, R. (2000) Tuberculosis Diagnosed during Pregnancy: A Prospective Study from London. Thorax, 55, 129-132. https://doi.org/10.1136/thorax.55.2.129

[14] Miller, K. and Miller, J. (1996) Tuberculosis in Pregnancy: Interactions, Diagnosis and Management. Clinical Obstetrics and Gynecology, 39, 120-142. https://doi.org/10.1097/00003081-199603000-00012

[15] World Health Organization (2015) Global Tuberculosis Report 2015. World Health Organization, Geneva.

[16] Awada, A. (1998) Evolution of Brain Tuberculomas under Standard Antituberculous Treatment. Journal of the Neurological Sciences, 156, 47-52. https://doi.org/10.1016/S0022-510X(98)00024-0

[17] Joint Tuberculosis Committee of the British Thoracic Society (1998) Chemotherapy and Management of Tuberculosis in the United Kingdom: Recommendations 1998. Thorax, 53, 536-548. https://doi.org/10.1136/thx.53.7.536

[18] Nahid, P., Dorman, S.E., Alipanah, N., Barry, P.M., Brozek, J.L., Cattamanchi, A., et al. (1987) Official American Thoracic Society/Centers for Disease Control and Prevention/Infectious Diseases Society of America Clinical Practice Guidelines: Treatment of Drug-Susceptible Tuberculosis. Clinurban Medical Center. Medicine (Baltimore), 66, 317-326.

[19] Poonnoose, S.I. and Rajshekar, V. (2003) Rate of Resolution of Histologically Verified Intracranial Tuberculoma. Neurosurgery, 53, 873-879.

https://doi.org/10.1227/01.NEU.0000083553.25421.6F 
[20] Rossi, L.N., Duzioni, N. and Terzi, F. (1985) Intracranial Tuberculoma in a Child: Regression on CT-Scan under Conservative Therapy. Neuropediatrics, 16, 228-230. https://doi.org/10.1055/s-2008-1059542

[21] Wahiba, G., Abdelkader, G., Houda, B., et al. (2018) Les tuberculomes cérébraux de l'adulte immunocompétent: 21 cas à Setif-Algerie. African Journal of Neurological Sciences, 37, 25-34. 